\title{
Dietary Factors Associated with Osteoporosis Among Post-Menopausal Women: A Study in a District Hospital, Jamalpur, Bangladesh
}

\author{
Fakhria Alam, ", Gulnar Begum², Farida Begum³, Md. Alauddin ${ }^{4}$ \\ ${ }^{1}$ Department of Gynaeocology \& Obstetrics, 250 Bedded General Hospital, Jamalpur, Bangladesh \\ ${ }^{2}$ Department of Radiology and Imaging, 250 Bedded General Hospital, Jamalpur, Bangladesh \\ ${ }^{3}$ Department of Gynaeocology \& Obstetrics, Sher E Bangla Medical College Hospital, Barishal, Bangladesh \\ ${ }^{4}$ Department of Anaesthesiology, Sherpur District Hospital, Mymensingh, Bangladesh
}

Email address:

fakhriaalam65@gmail.com (F. Alam)

${ }^{*}$ Corresponding author

\section{To cite this article:}

Fakhria Alam, Gulnar Begum, Farida Begum, Md. Alauddin. Dietary Factors Associated with Osteoporosis Among Post-Menopausal Women: A Study in a District Hospital, Jamalpur, Bangladesh. Biomedical Sciences. Vol. 7, No. 3, 2021, pp. 69-73.

doi: $10.11648 /$ j.bs.20210703.12

Received: April, 26, 2021; Accepted: May 31, 2021; Published: August 5, 2021

\begin{abstract}
Introduction: Osteoporosis is a bone disease that occurs when the body loses too much bone, makes too little bone, or both. As a result, bones become weak and may break from a fall or, in serious cases, from sneezing or minor bumps. The aim of the study was to assess dietary Factors Associated with Osteoporosis among Post-Menopausal Women. Material \& Methods: This was an analytical cross-sectional study at the department of Obstetrics \& Gynecology of 250 bedded General Hospital, Jamalpur, Bangladesh during the period from January 2018 to December 2018. Results: Out of 300 post-menopausal women the maximum number of respondents were from the age group of $45-55$ years $(42.33 \%)$ followed by $56-65$ years $(27.67 \%)$ and $>85$ years $(4.33 \%)$. The mean age of the respondents was $65.66 \pm 12.31$ years and the mean age at menopause was $47.35 \pm 3.78$ years. Maximum respondents were from overweight $(42.67 \%)$ whereas the least number of respondents were from underweight (1.67\%). The mean BMI of all respondents was 27.39 \pm 4.93 . Most of the patients experienced menopause for last 6-10 years $(63 \%)$ whereas the least number of patients experienced menopause for only $1-5$ years (11\%). $183(61 \%)$ respondents stated that they did not take any milk specific diet like milk, yogurt, sweetmeats, card etc. regularly whereas rest $117(39 \%)$ agreed that they took milk or dairy products in any form mentioned above. Among 300 respondents, it was observed that $45(15 \%)$ did not take tea in their day to day life while the number of habits of not taking coffee was $285(95 \%)$. Habit of taking tea was found in $255(85 \%)$ respondents and coffee having habit was found in case of $15(5 \%)$ respondents. Compared to the respondents who experienced menopause $>10$ years were suffered from osteoporosis 0.0751 times more in comparison to the respondents who experienced menopause for the duration of $\leq 10$ years, which is statistically highly significant at $5 \%$ level of significance (95\% CI: $0.0101-0.5582, \mathrm{p}=0.0012)$. Conclusion: Milk or milk related product regularly have less likely to have osteoporosis than those who do not take any of these daily. So, it was significant that post menopause women who takes milk daily have less risk of osteoporosis.
\end{abstract}

Keywords: Osteoporosis, Dietary Factors, Post-menopausal, Overweight

\section{Introduction}

Low trauma fractures are the most significant outcome of osteoporosis and occur most frequently at the hip, spine and distal forearm. Osteoporotic fractures occur as a consequence of a triad of factors: osteoporosis, falls and the interface/impact. Notably, the strongest single risk factor for fracture is falling. [1] Osteoporosis is often called a silent 
disease because bone loss occurs without symptoms. People may not know that they have osteoporosis until their bones become so weak that a sudden strain, bump, or fall causes a hip to fracture or a vertebra to collapse. Collapsed vertebrae may initially be felt or seen in the form of severe back pain, loss of height, or spinal deformities such as kyphosis (severely stooped posture) [2] Women are at increased osteoporosis risk related to estrogen levels if they: Experience irregular or infrequent periods, or began having their periods at a later than normal age, have had their ovaries removed (at any age), are going through menopause, with those undergoing menopause at an early age having an even higher risk, women loss bone mass much more quickly in the years immediately after menopause than they do at any other time in their lives. [3] Evidence suggests that childhood represents a window of opportunity to strengthen the skeleton and reinforces the need for health promotion initiatives to be directed towards this age group. Although physical activity during growth contributes to greater gains in bone accrual into young adulthood relative to less physical activity there remains insufficient evidence to conclude that exercise during growth prevents fragility fractures in late adulthood. This evidence gap largely reflects the difficulty in undertaking robust research to address this question. The importance of the in-utero period for influencing peak bone mass, particularly with respect to nutrition during fetal development is also recognized. Fracture risk is dependent not only on osteoporosis but also on exposure of the skeleton to trauma, in the older age groups most commonly as falls. Many of the risk factors for falls are modifiable and there is robust evidence for the effectiveness of multimodal interventions to prevent falls in people in the community and in care facilities. [4] Diet and lifestyle play a crucial role in maintaining the health of this growing portion of the community. It is commonly held view that food or a food component can offer medical or health benefits above and beyond simple nutrition. [5] A comprehensive osteoporosis treatment program includes a focus on proper nutrition, exercise, and safety issues to prevent falls that may result in fractures. In addition, doctor may prescribe a medication to slow or stop bone loss, increase bone density, and reduce fracture risk. Preventing falls is a special concern for men and women with osteoporosis. Falls can increase the likelihood of fracturing a bone in the hip, wrist, spine, or other part of the skeleton. Falls can also be caused by impaired vision or balance, chronic diseases that affect mental or physical functioning, and certain medications, such as sedatives and antidepressants. It is important that individuals with osteoporosis be aware of any physical changes that affect their balance or gait, and that they discuss these changes with their health care provider. [6]

\section{Objectives}

General objective

To assess the dietary factors associated with osteoporosis among post-menopausal women

\section{Specific Objectives}

To describe the health-related factors associated with osteoporosis among post-menopausal women

\section{Methods}

This was a cross-sectional study conducted at the department of Obstetrics \& Gynecology of 250 bedded General Hospital, Jamalpur, Bangladesh during the period from January 2018 to December 2018. Three hundred (300) clinically suspected female patients aged between 45 and 85 years were selected as study participants. Statistical software SPSS were used for data analysis.

\section{Inclusion Criteria}

Post-menopausal women who are willing to response.

Exclusion Criteria

Post-menopausal women who had experienced surgical or unnatural menopause, regardless of age.

\section{Results}

Out of 300 post-menopausal women the maximum number of respondents were from the age group of 45-55 years $(42.33 \%)$ followed by $56-65$ years $(27.67 \%)$ and $>85$ years $(4.33 \%)$. The mean age of the respondents was $65.66 \pm 12.31$ years and the mean age at menopause was $47.35 \pm 3.78$ years. Maximum respondents were from overweight (42.67\%) whereas the least number of respondents were from underweight (1.67\%). The mean BMI of all respondents was $27.39 \pm 4.93$. Most of the patients experienced menopause for last 6-10 years $(63 \%)$ whereas the least number of patients experienced menopause for only 1-5 years (11\%). According to the $\mathrm{t}$ score of bone mineral density of the respondent's osteoporosis found in case of 267 women (89\%), Osteopenia found in $23(7.6 \%)$ and normal density found in $10(3.33 \%)$ women. A mass number of the respondents in this study came from average middle- and low-income population. Majority of the respondents $(77 \%)$ did not undergo regular exercise whereas rest $23 \%$ underwent this procedure. $183(61 \%)$ respondents stated that they did not take any milk specific diet like milk, yogurt, sweetmeats, card etc. regularly whereas rest 117 (39\%) agreed that they took milk or dairy products in any form mentioned above. Among 300 respondents, it was observed that $45(15 \%)$ did not take tea in their day to day life while the number of habits of not taking coffee was 285 (95\%). Habit of taking tea was found in 255 $(85 \%)$ respondents and coffee having habit was found in case of $15(5 \%)$ respondents. Out of 255 respondents of having tea $20(7.84 \%)$ took 1 cup/day $75(29.41 \%)$ took 2 cup/day and $160(62.74 \%)$ took 3 cup/day whereas of out of 15 respond of $6(40 \%), 5(29.41 \%)$ and $4(26.67 \%)$ took $1,2,3$ cup coffee per day respectively. $95.67 \%$ took no medication among our study population but only a few women took corticosteroids $(3.33 \%)$ and anti-depressive drugs (1\%). In this study, no women experienced any gastrointestinal surgery. Maximum patients underwent different bone fractures (77\%) (radius, ulna, tibia-fibula, humerus, femur, ribs, skull, scapula, 
clavicle) other than hip (2\%), spinal $(5 \%)$ and wrist $(4 \%)$ fractures. Only $12 \%$ of the total respondents didn't experience any fracture. Compared to respondents with age less than 65, respondents with age group more than 65 have 0.090 times more likely to have prevalence of osteoporosis, which is statistically highly significant at $5 \%$ level of significance (95\% CI: 0.0215-0.3844, $\mathrm{p}<0.05)$. Compared to the respondents who experienced menopause $>10$ years were suffered from osteoporosis 0.0751 times more in comparison to the respondents who experienced menopause for the duration of $\leq 10$ years, which is statistically highly significant at $5 \%$ level of significance (95\% CI: 0.0101-0.5582, $\mathrm{p}=0.0012$ ).

Table 1. Distribution of the respondents by Age $(n=300)$.

\begin{tabular}{lllll}
\hline Age of respondents & Frequency $(\mathbf{n}=\mathbf{3 0 0})$ & Percentage $(\mathbf{\%})$ & Mean \pm SD years & Mean Age at menopause \\
\hline $45-55$ & 127 & 42.33 & & \\
$56-65$ & 83 & 27.67 & $65.66 \pm 12.31$ & $47.35 \pm 3.78$ \\
$66-75$ & 40 & 13.33 & & \\
$76-85$ & 37 & 1.33 & & \\
$>85$ & 13 & 4.33 & & \\
\hline
\end{tabular}

Table 2. Distribution of BMI of the respondents and Mean of BMI \& Weight ( $n=300)$.

\begin{tabular}{lllll}
\hline BMI of the respondents & Frequency & Percentages (\%) & Mean \pm SD BMI & Mean \pm SD weight \\
\hline$<18.5$ (underweight) & 5 & 1.67 & & \\
$18.5-24.9$ (normal) & 107 & 35.67 & $27.39 \pm 4.93$ & $61.76 \pm 0.18$ \\
$25-29.9$ (overweight) & 128 & 42.67 & & \\
$>30$ (obesity) & 60 & 20 & & \\
\hline
\end{tabular}

Table 3. Distribution of the respondents according to duration of menopause ( $n=300)$.

\begin{tabular}{llll}
\hline Duration (Years) & $\mathbf{N}$ & $\%$ & \\
\hline $1-5$ & 33 & 11 & \\
$6-10$ & 189 & 63 & 26 \\
$>10$ & 78 & 26 \\
\hline
\end{tabular}

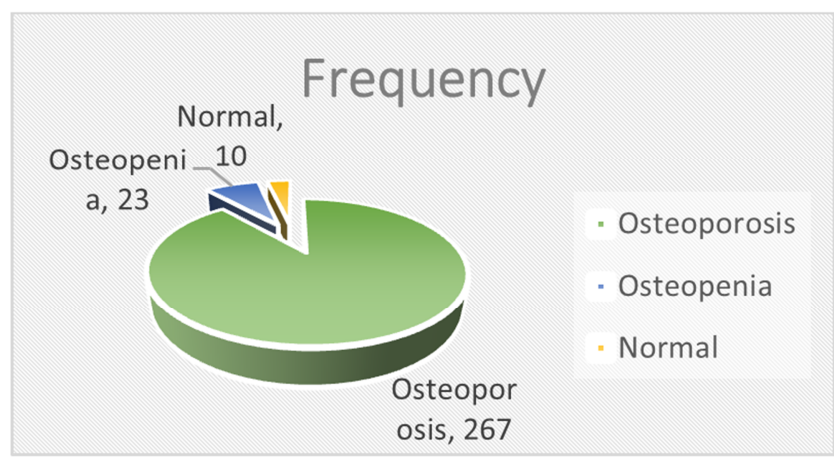

Figure 1. Distribution of BMD of the respondents $(n=300)$.

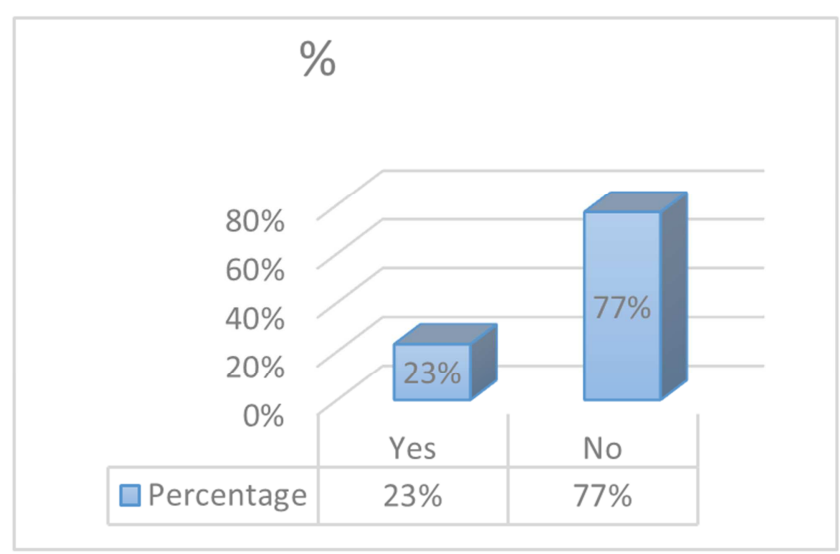

Figure 2. Distribution of respondents by Physical exercise $(n=300)$.

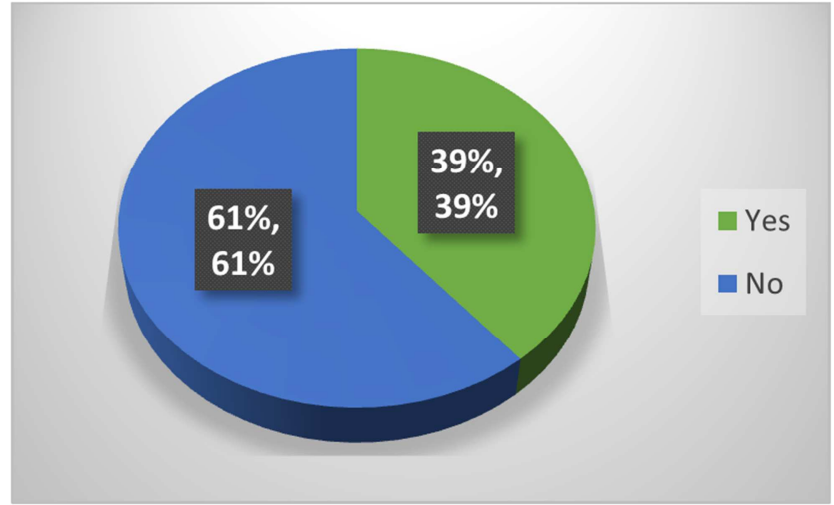

Figure 3. Distribution of the respondents by taking dairy product like milk/yogurt $(n=300)$.

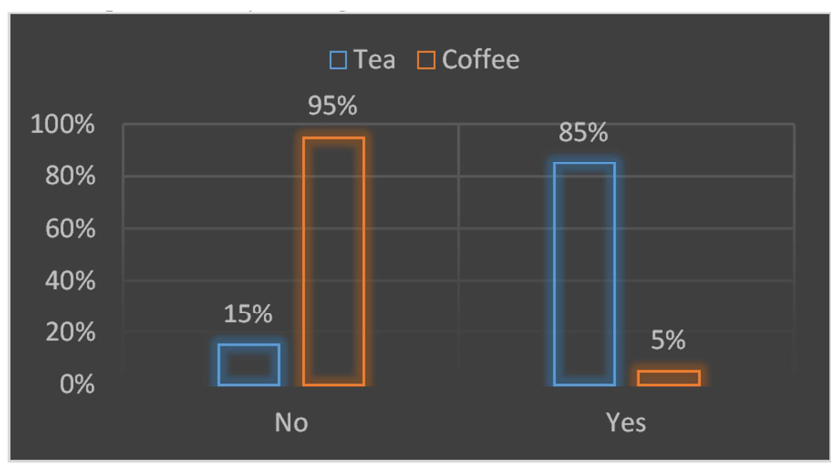

Figure 4. Distribution of the respondents by taking tea and coffee. $(n=300)$. 

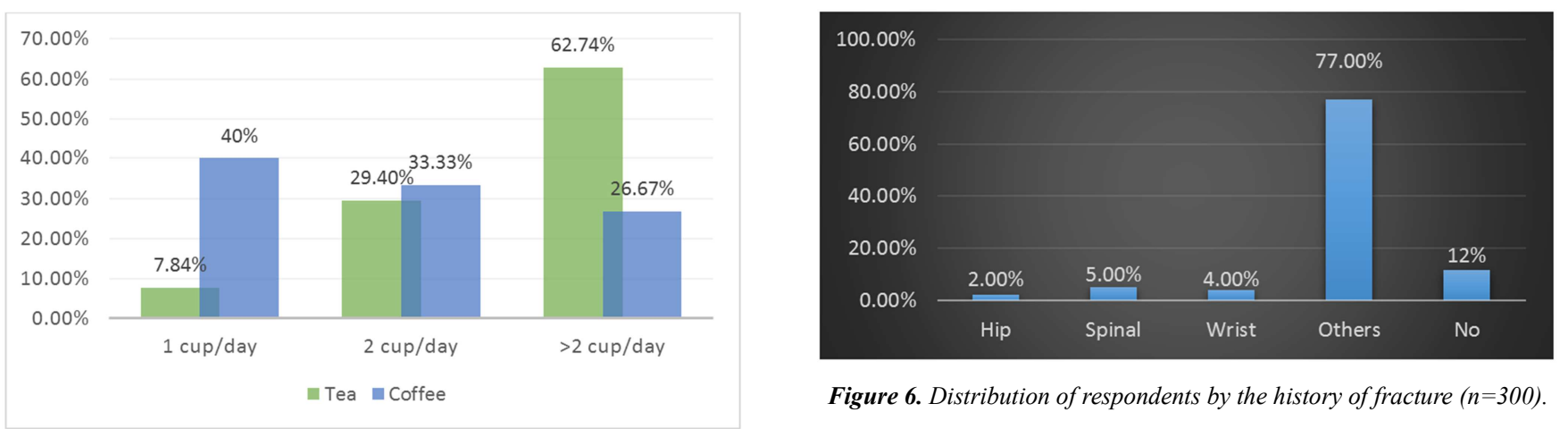

Figure 6. Distribution of respondents by the history of fracture $(n=300)$.

Figure 5. Distribution of the respondents by the amount of drinking tea, coffee $(n=300)$.

Table 4. Distribution of the respondents by Health Measures ( $n=300)$.

\begin{tabular}{lll}
\hline Health related factors & $\mathbf{N}=\mathbf{3 0 0}$ & $\mathbf{\%}$ \\
\hline Medication taken & & $3.33 \%$ \\
Corticosteroid for three months & 10 & $1 \%$ \\
Anti-depressive drug & 3 & $95.67 \%$ \\
None & 287 & $0 \%$ \\
Done gastro intestinal surgery & & 100 \\
Yes & 0 & \\
No & 300 & \\
\hline
\end{tabular}

Table 5. Cross tabulation between age and bone mineral density $(n=300)$.

\begin{tabular}{|c|c|c|c|c|}
\hline \multirow[t]{2}{*}{ Age } & \multicolumn{2}{|c|}{ Bone mineral Density } & \multirow{2}{*}{ OR $(95 \% \mathrm{CI})$} & \multirow{2}{*}{ P value } \\
\hline & Osteoporosis & Normal & & \\
\hline $\begin{array}{l}<65 \text { (ref) } \\
>65\end{array}$ & $\begin{array}{l}168(94.28 \%) \\
88(97.78 \%)\end{array}$ & $\begin{array}{l}42(5.71 \%) \\
2(2.22 \%)\end{array}$ & $0.090(0.0215-0.3844)$ & $<0.05 \mathrm{~S}$ \\
\hline
\end{tabular}

Table 6. Cross tabulation between duration of menopause and bone mineral density $(n=300)$.

\begin{tabular}{lllll}
\hline Duration of menopause & Bone mineral Density & \multirow{2}{*}{ OR (95\% CI) } & \multirow{2}{*}{ P value } \\
\hline & Osteoporosis & Normal & \multirow{2}{*}{$0.0751(0.0101-0.5582)$} & $0.0012^{\mathrm{S}}$ \\
\hline$\leq 10$ years (ref) & $192(84.21 \%)$ & $36(15.78 \%)$ & $1(1.23 \%)$ & $0.07 \%)$ \\
\hline 10 years & $71(98.61 \%))$ & & &
\end{tabular}

\section{Discussion}

Among the participants $45-55$ years were $42.33 \%$ and $27.67 \%$ were between the ages of 56-65 years. There is significant correlation between bone mineral density and age $(\mathrm{p}=<0.05)$, which showed that osteoporosis increased with advancing age. Compared to respondent with age less than 65 , patients with age more than 65 years is 0.090 times more likely to have prevalence of osteoporosis. There is significant correlation between bone mineral density and age $(\mathrm{p}=0.05)$. Our findings demonstrated a significant correlation between age and osteoporosis. As the age increases, osteoporotic cases increase, especially after 65 years, this is consistent with other studies where osteoporosis is prevalent in women after 60. Several reasons were reported age as a risk factor due to estrogen deficiency after menopause. [7, 8] Among the interviewed respondents, $42.67 \%$ were overweight with the BMI of less than 25. Although, $35.67 \%$ of the respondents had normal BMI (18.5-24.9), and minority (1.67\%) was underweight. The different study says that overweight BMI has high chance osteoporosis. [10] Our study also shows that overweight patients has 0.0637 times more chances to have osteoporosis than underweight patients and the result is statistically significant. In this study, $98.61 \%$ of respondents had duration of menopause more than 10 years. Respondents having duration of menopause years less than or equal to 10 years have $84.21 \%$ less chances to have osteoporosis than respondents with menopause years more than 10 years $(\mathrm{OR}=0.0751 ; 95 \% \mathrm{CI}=0.0101-0.5582 ; \mathrm{p}=0.0012)$. Similar to the study where bone loss was significantly related number of years since menopause. [9] According to WHO criteria, we observed in our study that, $89 \%$ of respondents had Osteoporosis, $7.66 \%$ were osteopenia and 3.33\% had Normal BMD at wrist site. In women of Delhi India, prevalence of osteoporosis was $42.5 \%$ and osteopenia $44.9 \%$. [10] In premenopausal Dutch women the prevalence of osteopenia was $27.3 \%$ and $4.1 \%$ of the women were osteoporotic. [11] The study conducted in 581 postmenopausal white women to examine the relationship between lifetime milk consumption both as axial and appendicular bone mineral density shows that, regular milk consumption in youth and adulthood is associated with better bone mineral density at cortical and trabecular sites in elderly women. [12] Similarly, the study in Korea suggested that high consumption of milk, dairy products, and green tea 
may reduce the risk of osteoporosis in postmenopausal Korean women. [13] In our study $39 \%$ of the total respondent stated that they take milk or milk containing product daily. Whereas $61 \%$ stated that they do not take any milk or milk related products. So, respondent who take milk or milk related product regularly have less likely to have osteoporosis than who do not take any of these daily. A cohort study of 31,527 Swedish women aged 4076 years at baseline in 1988 that shows, the consumption of coffee, caffeinated tea and the intake of caffeine were estimated from a self-administered food frequency questionnaire (FFQ). Which indicate that a daily intake of $330 \mathrm{mg}$ of caffeine, equivalent to 4 cups $(600 \mathrm{ml})$ of coffee or tea, or more may be associated with a modestly increased risk of osteoporotic fractures, especially in women with a low intake of calcium. [14] In our study $44.33 \%$ of the respondents take tea more than 2 cups per day whereas, $26.33 \%$ stated that they take less than 2 cup per day. So, taking tea 2 cups or more than 2 cups has significant association with osteoporosis. The history of previous fracture was associated with osteoporosis, in this study the respondents had $64.33 \%$ history of fracture due to osteoporosis. Similarly, in a survey of 2649 ambulatory postmenopausal women using single-photon absorptiometry of the forearm and a detailed questionnaire on falls and fractures it was found that falls are a major contribution factor to the occurrence of symptomatic fractures in postmenopausal women, independent of and additive to the risk attributable to age and osteoporosis. [15] The launch of the WHO technical report, assessment of osteoporosis at primary health care level, and the related web based FRAX tool are the major milestones toward helping health professionals worldwide to improve identification of patients at high risk of fractures. [16] A risk assessment tool for osteoporosis developed by Sharma and Khandelwal can be effective in a resource poor nation like India, where they used a combination of questionnaire and ultrasonic measurement of BMD. Although DEXA scan is considered as a gold standard for BMD assessment, most of the Subcontinent women cannot afford it due to the cost involved. [17]

Limitations of the study

This was a prospective type of study in a single community with comparatively small number of sample size. So, the study result may not reflect the exact scenarios of the whole country.

\section{Conclusion}

Menopause is highly responsible for osteoporosis. Other high-risk factors associated with low BMD were found to be low BMI, low dietary calcium intake, lack of exercise, and increasing age. It was significant that, post menopause women who takes milk daily have less risk of osteoporosis. There was a significant positive correlation between increasing age, low BMI, low calcium intake, lack of exercise, and low BMD. Thus, high prevalence of osteoporosis in postmenopausal women is a major health concern. Although no symptoms occur prior to fracture, BMD and other risk factors can be used to identify high risk patients, and because effective interventions exist, many of these fractures are now preventable.

\section{References}

[1] Osteoporosis in menopausal women in Nepal- Google search. Available from URL: http://www.google.com.np.

[2] Lane JM, Serota AC, Raphael B: Osteoporosis: Differences and Similarities in Male and Female Patients. Orthop Clin N Am 37 2006; 601-609.

[3] Estrogen. http://en.wikipedia.org/wiki/Estrogen.

[4] International Osteoporosis Foundation; The Asian Audit: epidemiology, costs and burden of osteoporosis in Asia 2009. http://www.iofbonehealth.org/sites/default/files/PDFs/Audit\% 20Asia/Asian_regional_audit_India.pdf.

[5] Nahim, R. L. and S. E. Straus, 2001, Research into complementary and alternative medicine: Problems and potential. BMJ, 322: 161-164.

[6] Consensus Development Conference. Diagnosis, prophylaxis and treatment of osteoporosis. Am J. Med. 1993; 94: 646-50.

[7] Li S et al. The correlation of osteoporosis to clinical features: a study of 4382 female cases of a hospital cohort with musculoskeletal symptoms in southwest China. BMC Musculoskeletal Disorders, 2010, 11: 183-192 (29).

[8] Bjarnason $\mathrm{NH}$ et al. Number of years since menopause: spontaneous bone loss is dependent but response to hormone replacement therapy is independent enter for Clinical and Basic Research, Ballerup, Denmark.

[9] Svejme O et al Phsical activity reduces bone loss in the distal forearm in post-menopausal women-a 25 -year prospective study.

[10] Micklesfield LK et al. Lifestyle questionnaire to evaluate risk for reduced bone mineral density in women. Clin J Sport Med. 2005; 15 : 340-348.

[11] Kanis JA et al. Risk of ip fracture according to the World Health Organization criteria for osteopenia and osteoporosis. Bone 2000, 27: 585-590 (6).

[12] Soroko S, Holbrook TL, Edelstein S, Barrett-Connor E (1994) Milk consumption and bone mineral density in older women: The Rancho Bernardo Study. Am J Public Health 84: 1319-1322.

[13] Park SJ et al. Dietary patterns and osteoporosis risk in postmenopausal Korean women song 2012 Dec; 3 (4): 199205. doi: 10.1016/j-phrp. 2012.10.005.

[14] Hall strom et al Coffee, tea and caffeine consumption in relation to osteoporotic fracture risk in a cohort of Swedish women. Osteoporosis (2006) 17: 1055-1064.

[15] Lau EM et al Bone mineral density in Chinese elderly female vegetarians, vegans, lacto-vegetarians and omivores. Department of Community and Family Medicine, Chinese University of Hong Kong.

[16] Kanis JA. On behalf of the world health organization scientific group. Assessment of osteoporosis at the primary healthcare level. WHO Collaborating Centre for Metabolic Bone Diseases. England, UK: University of Sheffield; 2008.

[17] Sharma S, Khandelwal S. Effective risk assessment tools for osteoporosis in the Indian menopausal female. J Mid Life Health 2010; 1: 79-85. 